\title{
Les moulins à vent de Basse-Normandie
}

\section{Benoît Canu}

\section{OpenEdition \\ Journals}

\section{Édition électronique}

URL : https://journals.openedition.org/adlfi/3803

ISSN : 2114-0502

Éditeur

Ministère de la Culture

Référence électronique

Benoît Canu, «Les moulins à vent de Basse-Normandie » [notice archéologique], ADLFI. Archéologie de la France - Informations [En ligne], Normandie, mis en ligne le 01 mars 2009, consulté le 22 juin 2021.

URL : http://journals.openedition.org/adlfi/3803

Ce document a été généré automatiquement le 22 juin 2021.

(c) ministère de la Culture et de la Communication, CNRS 


\title{
Les moulins à vent de Basse- Normandie
}

\author{
Benoît Canu
}

Date de l'opération : 2009 (PT)

1 En dépit d'un intérêt avéré et de l'utilité d'accorder au débat sur les énergies une profondeur historique, les moulins à vent de Basse-Normandie n'ont suscité qu'une attention modérée, bien moindre que leurs confrères ou concurrents hydrauliques certes mieux documentés. D'abord concentrées sur le Cotentin, désormais étendues à l'ensemble du Calvados et de la Manche (îles anglo-normandes incluses) et bientôt à l'Orne, nos recherches ont d'ores et déjà permis de recenser un nombre de places et une diversité d'usages supérieurs à ce que les enquêtes industrielles, la carte de Cassini ou des inventaires informels laissaient entrevoir. Ainsi, aux côtés d'une majorité d'ateliers voués à la trituration des grains, la lecture de sources écrites a permis d'exhumer des unités spécialisées dans l'extraction d'huile, la préparation du pastel, le sciage du bois, la pulvérisation de la pierre, le broyage des carcasses animales ou encore l'élévation de l'eau.

2 L'examen comparé d'archives cadastrales et de cartes à grande comme à petite échelle a surtout permis de situer, à ce jour, près de 400 moulins à vent et de confirmer un déséquilibre dont témoignent une micro-toponymie très conservatrice et, dans une moindre mesure, la soixantaine d'unités conservées : l'Orne en compterait trois, isolées dans le Grand Perche, le Calvados une dizaine, la plupart en Bessin, la Manche une cinquantaine dont quarante pour le seul Cotentin ; cinq subsisteraient à Guernesey, deux ou trois à Jersey comme à Serk. Si les trois quarts des ateliers restants ponctuent donc, du Mont-Saint-Michel à la Hague, la côte ouest de la Manche et les îles et coteaux du Seuil du Cotentin, en revanche, le plateau du Bessin mais surtout les plaines de Caen, Falaise ou Sées ne témoignent plus guère ou plus du tout du semis relativement dense qui animait autrefois leurs campagnes.

3 Une raison majeure de ces disparitions pourrait tenir à la prépondérance d'un type fait essentiellement de bois, commun au nord de la Loire, majoritaire même en Haute- 
Normandie. Orientable face au vent, la cabine de ce moulin dit "à pivot fixe » était perchée sur un axe de forte section, solidement étayé. Mais alors que les soles qui reçoivent ces étais sont fréquemment englobées dans une construction basse (la cavette), elles pourraient ici, à l'instar des moulins du Roumois et du Pays de Caux, n'avoir été que posées sur des dés superficiellement fondés, susceptibles de n'avoir laissé qu'une empreinte archéologique ténue. Connu à l'est de la région, attesté à Granville et pressenti plus au nord, la répartition géographique du «pivot fixe » basnormand autant que ses particularismes mériteraient donc d'être précisés. Il conviendrait aussi de voir dans quelle mesure, dans le cadre des reconstructions de la guerre de Cent Ans par exemple, des tours ou tourelles des modèles suivants purent lui être substituées.

En l'absence de "pivots fixes ", la plupart des vestiges conservés relèvent en effet de classiques «moulins-tours ", tels qu'il s'en visite dans la Manche. Seule la charpente de toit, porteuse de l'arbre moteur, virait au moyen d'un timon. Abritant trois voire quatre niveaux, appareillées en pierres locales (à une exception près, faite de bauge), ces «tours ou masses» sont réputées n'avoir pas profité d'améliorations mécaniques présumées trop lointaines ou, au regard d'une déchéance précoce, trop tardives. Toutefois, l'adoption de ces progrès étant attestée aux frontières de la région, le degré de modernisation mériterait d'être reconsidéré. Au-delà de ces préoccupations techniques, il serait également utile de préciser leurs nuances architecturales et vérifier ou proposer des critères typologiques de datation.

Enfin, investigations documentaires et enquêtes de terrain ont enrichi le corpus et la connaissance d'un troisième type, propre à l'Ouest et, en Normandie, au Bessin et au Cotentin. De loin le plus original et le moins connu, sa compréhension pâtit d'un nom énigmatique - «turquois » - d'origines obscures ainsi que de préjugés tenaces dus à la disparition des éléments supérieurs. En cours de relevés, leurs restes ne consistent qu'en une masse tronconique ou cylindrique, simple ou à ressauts, mais toujours d'une qualité remarquable, conforme à leur statut. Percée d'un conduit vertical dégressif, d'une porte et de niches ou soupiraux latéraux, elle enserrait une sole et un arbre porteur mobile, solidaire de la cabine de travail ; on parle ainsi de moulin à " pivot tournant ». Or, la mise à jour des deux seules photographies connues d'un spécimen " en état " suggère une charpente parfaitement atypique, comme "inversée ". Disposition jusqu'alors seulement attestée au nord de la Baltique mais suggérée déjà sur le tableau d'un moulin des bords de Loire, l'arbre moteur s'y trouverait non à hauteur de comble mais au bas de la cage, entrainant les meules par-dessous, à la façon d'un moulin à eau. Alors possible chaînon manquant dans l'histoire des techniques, ce type reste visible en Normandie en une dizaine de places dont celle occupée dès avant 1180 par l'un des tout premiers moulins à vent cités en Occident. La quête de compléments descriptifs, une réinterprétation du qualificatif «turquois » autant que des fouilles ou l'analyse de mortiers sont donc attendues pour asseoir la portée de ces découvertes et espérer enfin définir les traits d'une " architecture-machine » dont l'intérêt confirmé réclame plus que jamais une attention particulière.

6 (Fig. $\mathrm{n}^{\circ} 1$ : Moulins-tours de Bavent (Sénoville - 50) et Gouville (50);moulins à pivot tournant du Mesnil (Saint-Germain-de-Varreville - 50) et de Saint-Jean (Le Vicel - 50), plans et vues en élévations) et (Fig. $n^{\circ} 2$ : Carte postale ancienne conservée aux Archives départementales de la Manche)

Benoît CANU 


\section{ANNEXES}

Fig. $\mathrm{n}^{\circ} 1$ : Moulins-tours de Bavent (Sénoville - 50 ) et Gouville $(50)$;moulins à pivot tournant du Mesnil (Saint-Germain-de-Varreville - 50) et de Saint-Jean (Le Vicel - 50), plans et vues en élévations

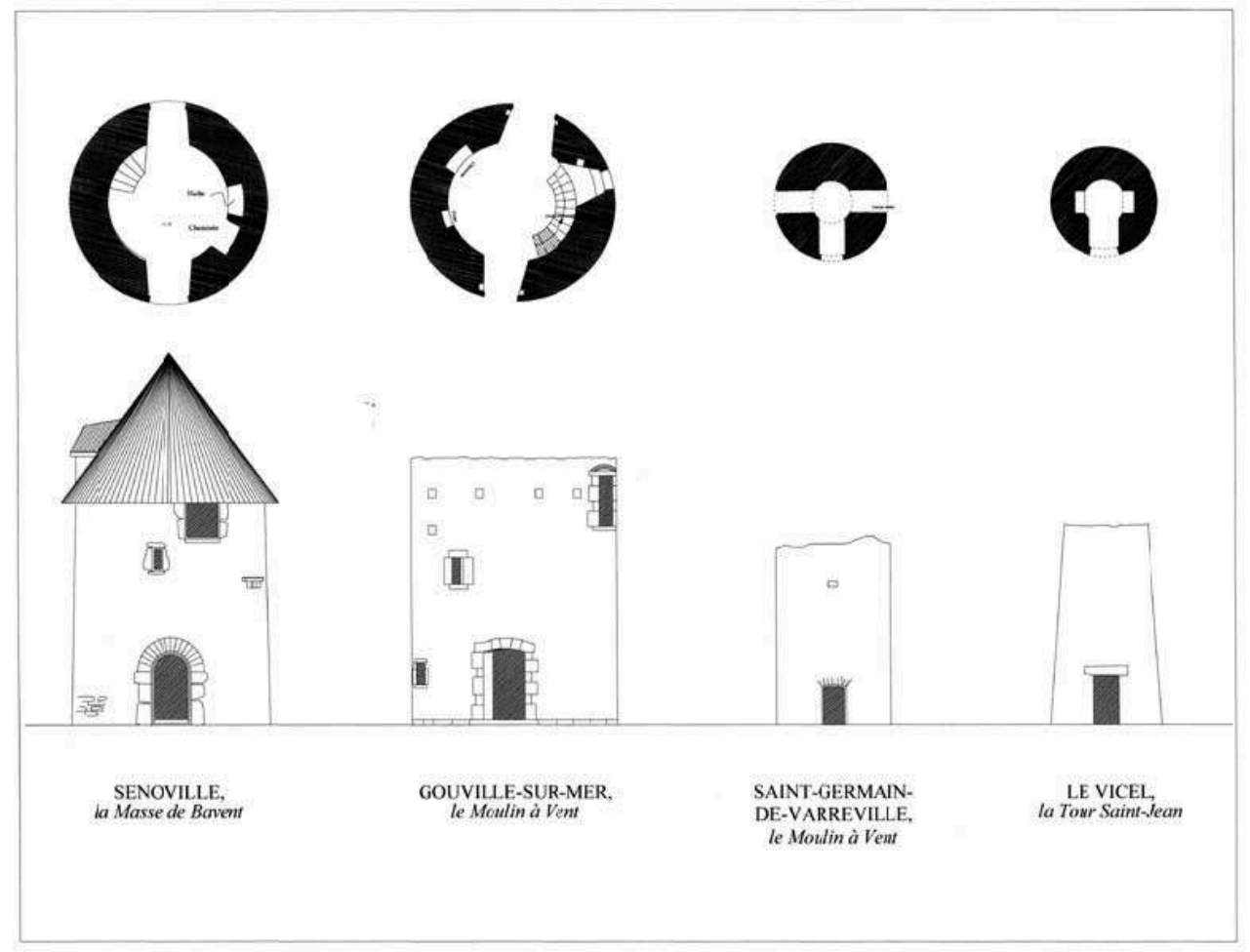

L. Lamotte ; B. Canu (2009) 
Fig. $\mathrm{n}^{\circ} 2$ : Carte postale ancienne conservée aux Archives départementales de la Manche

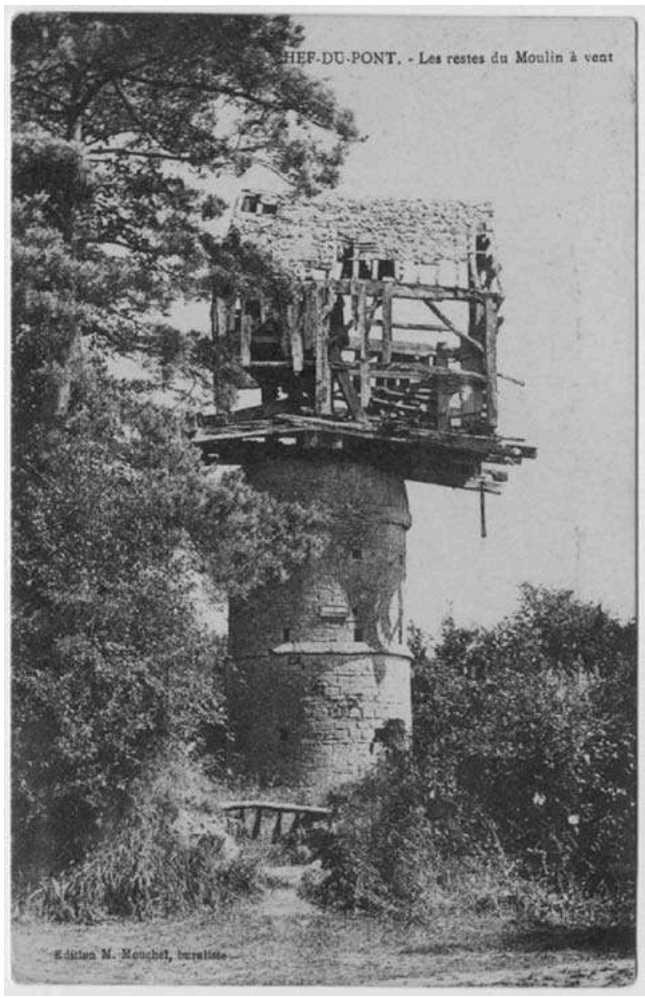

Archives départementales de la Manche (2009)

\section{AUTEURS}

BENOÎT CANU

BEN 